\title{
GESTIÓN DE CONOCIMIENTO APLICADO AL GRUPO BIBLIOTECAS E INFORMACIÓN UPTC
}

\section{APPLIED KNOWLEDGE MANAGEMENT GROUP LIBRARY AND INFORMATION UPTC}

\author{
Tatiana Blanco Rojas* y Diana Milena Archila Córdoba**
}

\begin{abstract}
RESUMEN
Viendo el gran significado que tiene la información organizada en la era actual y la necesidad de comunicarla de forma adecuada, la presente investigación se realiza con el fin de estudiar estrategias para implementar un sistema de gestión del conocimiento en el Grupo de Bibliotecas e Información de la Universidad Pedagógica y Tecnológica de Colombia (UPTC)- seccional Tunja; para esto se evaluarán tres aspectos importantes del conocimiento: la novedad, la transformación y la difusión, los cuales son indispensables en cualquier área de trabajo para lograr que todos los procesos marchen correctamente.
\end{abstract}

Palabras clave: Gestión del conocimiento, Biblioteca, Novedad, Transformación, Difusión.

\begin{abstract}
Seeing the great significance of the information organized in the current era and the need to communicate properly, this research is conducted in order to explore strategies to implement a knowledge management system in the group of libraries and information of Universidad Pedagógica y Tecnológica de Colombia (UPTC)- sectional Tunja, to assess this knowledge three important aspects: the development, processing and dissemination, which are essential in any work area to ensure that all processes properly march.
\end{abstract}

Keywords: Knowledge Management, Library, Novelty, Processing, Dissemination.

Fecha de recepción: Mayo 28 de 2014 / Fecha de aceptación: Septiembre 23 de 2014

Tipología: Artículo de Reporte de Caso

Para citar el artículo: Blanco, R. T., \& Archila, C. D. (2014). Gestión de conocimiento aplicado al grupo: bibliotecas e información UPTC. Praxis, Vol. 10, 146 - 164

*Ingeniera de Sistemas y Computación de la Universidad Pedagógica y Tecnológica de Colombia, Colombia. E-mail: tatiana.blanco@uptc.edu.co.

**Ingeniera de Sistemas y Computación de la Universidad Pedagógica y Tecnológica de Colombia, Colombia. E-mail: diana.archila@uptc.edu.co. 


\section{INTRODUCCIÓN}

$\mathrm{D}$ ía tras día se generan nuevos conocimientos que deben ser gestionados y organizados para que el mundo entero pueda saber de ellos, por esta razón dentro de este reporte de caso se busca estudiar estrategias para implementar un sistema de gestión del conocimiento en el Grupo de Bibliotecas e Información de la UPTC seccional Tunja. Por otra parte, en la Tabla 1, se encuentran los antecedentes de la investigación con el fin de entender cómo surge el conocimiento, cómo avanza y cómo se aplica al caso de estudio (las bibliotecas).

\section{Tabla 1}

Antecedentes de la investigación

\begin{tabular}{|c|c|}
\hline Autor & Investigación \\
\hline (Bonnet, 2005) & $\begin{array}{l}\text { La Gestión del conocimiento aplicado a los } \\
\text { recursos de bibliotecas públicas }\end{array}$ \\
\hline (Davenport \& Prusak, 2001) & $\begin{array}{l}\text { Conocimiento en acción: como las organiza- } \\
\text { ciones manejan lo que saben. }\end{array}$ \\
\hline (Drucker \& Toffler, 2011) & $\begin{array}{l}\text { Gestión del conocimiento: contexto y } \\
\text { antecedentes }\end{array}$ \\
\hline (Gairín, 2007) & $\begin{array}{l}\text { Competencias para la gestión del conocimiento } \\
\text { y el aprendizaje }\end{array}$ \\
\hline (Gómez, 2007) & $\begin{array}{l}\text { Modelos para la creación y gestión del conoci- } \\
\text { miento: una aproximación teórica. }\end{array}$ \\
\hline (López, 2010) & $\begin{array}{l}\text { Grupo de Bibliotecas UPTC información } \\
\text { general }\end{array}$ \\
\hline $\begin{array}{l}\text { (Verde E., Blanco, C., \& Mata, } \\
\text { E., 2005). }\end{array}$ & $\begin{array}{l}\text { Gestión del conocimiento como fuente innova- } \\
\text { dora para las bibliotecas del sector universitario }\end{array}$ \\
\hline (Polanyi, M., \& Sen, A., 1996) & The Tacit Dimension \\
\hline (Hernández et al., 2008) & Metodología de la Investigación \\
\hline (Sánchez, 2007) & $\begin{array}{l}\text { Análisis de la gestión del conocimiento en las } \\
\text { bibliotecas universitarias de la Comunidad } \\
\text { Valenciana: propuesta de un modelo y acciones } \\
\text { de mejora. }\end{array}$ \\
\hline (Sandoval, 2013) & $\begin{array}{l}\text { Propuesta para implementar un sistema de } \\
\text { gestión del conocimiento que apoye el diseño } \\
\text { de un curso online. }\end{array}$ \\
\hline (Shanhong, 2000) & $\begin{array}{l}\text { Gestión del conocimiento en las bibliotecas } \\
\text { del siglo XXI }\end{array}$ \\
\hline (Tardón, 1999) & $\begin{array}{l}\text { Bibliotecas universitarias y gestión del } \\
\text { conocimiento }\end{array}$ \\
\hline (Trespalacios et al., 2005) & $\begin{array}{l}\text { Investigación de mercados: métodos de reco- } \\
\text { gida y análisis de la información para la toma } \\
\text { de decisiones en marketing. }\end{array}$ \\
\hline (UPTC, 2005) & $\begin{array}{l}\text { Estatuto General Universidad Pedagógica y } \\
\text { Tecnológica de Colombia. }\end{array}$ \\
\hline (Villamizar \& Weffer, 2011) & $\begin{array}{l}\text { Gestión del conocimiento en institutos univer- } \\
\text { sitarios de tecnología }\end{array}$ \\
\hline
\end{tabular}

Fuente: Autores 
El mundo gira alrededor de diversas fuentes económicas que sostienen a los seres humanos dentro de las cuales se conoce la tierra, el trabajo y el capital. En la sociedad actual ha surgido un nuevo tipo de economía denominada la economía o sociedad del conocimiento.

Ésta se distingue de las demás fuentes económicas porque en ella el conocimiento desempeña un papel esencial. Esta nueva economía no es solo otro recurso además de los tradicionales factores de producción, sino que es un capital valioso, útil y transmisible a quienes quieran acceder a él (Drucker \& Toffler, 2011).

Por otro lado, el conocimiento es la fuente del poder de más alta calidad y la clave del cambio, se ha convertido en la esencia del poder y es por eso que en todo el mundo se hace más ardua la batalla por controlar el conocimiento y los medios de comunicación (Drucker \& Toffler, 2011).

Pero hablando como tal de la gestión del conocimiento, ésta consiste en un conjunto de procesos sistemáticos orientados al desarrollo organizacional y/o personal y consecuentemente a la generación de una ventaja competitiva para la organización y/o el individuo (Gómez, 2007). En los últimos años esta gestión del conocimiento ha tomado una fuerza impresionante dentro de las pequeñas, medianas y grandes empresas que ven allí una fuente de capital importante y un cambio positivo de las organizaciones. El proceso de gestión del conocimiento consiste en mejorar la calidad de las decisiones que se toman en las organizaciones, esto se logra gracias a que se dispone de información fiable y válida que es entregada de manera oportuna y segura (Sandoval, 2013). La gestión del conocimiento implica transferencia personalizada para promover los intercambios espontáneos, las estrategias de transferencia del conocimiento además de preocuparse por la calidad, los contenidos, las herramientas informáticas y las comunicaciones que lo soportan, adicionalmente deben poner atención en las variables humanas que facilitan o bloquean la experiencia de compartir el conocimiento (Villamizar \& Weffer, 2011).
Un ejemplo de organizaciones promotoras del conocimiento son las bibliotecas que en sí mismas almacenan miles de referencias y documentos los cuales se pueden identificar, consultar y compartir para adquirir conocimiento tácito y explícito que posteriormente se podrá transmitir de maneras formales o informales. Las bibliotecas son gestoras de conocimiento, teniendo en cuenta que la gestión se refiere a identificar, adquirir, desarrollar, resolver, usar, almacenar y participar del conocimiento de una manera efectiva, a crear un acercamiento para transformar y compartir el conocimiento tácito y explícito y a aumentar la necesidad y capacidad de novedad utilizando el saber (Shanhong, 2000). Tanto la información como el conocimiento tienen que ver con las personas, pero en diferentes niveles o dimensiones (Bonnet, 2005), en donde a medida que se adquiere el conocimiento éste se transforma en creencias, conceptos y modelos mentales mediante el razonamiento y la reflexión.

Por otro lado, es necesario distinguir dos (2) clases de conocimiento: tácito y conocimiento explicito. El conocimiento tácito se refiere a un conocimiento que no puede articularse con facilidad y que por lo tanto, sólo existe en las manos y en las mentes de las personas, para manifestarse después mediante acciones. De otra parte, el conocimiento explícito es el conocimiento capturado y codificado en manuales, normas y procedimientos, resultando más fácil de transmitir (Polanyi \& Sen, 1996).

A las organizaciones les cuesta mucho explotar el conocimiento tácito al residir en el interior de las personas, pues incorpora tanto aprendizaje acumulado e inmerso que tal vez resulte imposible separar sus normas de la forma de actuar de un individuo (Davenport \& Prusak, 2001), por eso dentro del enfoque de esta investigación es importante desarrollar las competencias profesionales de los funcionarios y becarios que forman parte del Grupo de Bibliotecas e Información de la UPTC lo que determinará en gran medida la efectiva gestión del conocimiento en esta organización. 
Los objetivos de la gestión del conocimiento en las bibliotecas universitarias deben ir íntimamente ligados a los objetivos estratégicos de la propia universidad (Sánchez, 2007), vinculándolos en los planes interinstitucionales, en el contexto del plan estratégico, en la docencia y en la investigación. La gestión activa del conocimiento implica la construcción de procedimientos objetivos para convertir el conocimiento individual en patrimonio de la organización (Tardón, 1999). El eficaz manejo de la información es determinante en la excelencia académica dentro de las universidades, por esto, la competitividad depende de la calidad del servicio y de la toma de decisiones dentro de las organizaciones (Verde et al., 2005).

La Universidad Pedagógica y Tecnológica de Colombia (UPTC) es un ente universitario autónomo, de carácter nacional, estatal y público, democrático, de régimen especial, vinculado al Ministerio de Educación Nacional en lo referente a las políticas y la planeación del sector educativo, con sedes seccionales en Duitama, Sogamoso y Chiquinquirá, y con domicilio en Tunja (UPTC, 2005), cuenta con un Grupo de Bibliotecas e Información que internamente maneja un amplio conocimiento organizado de diversas maneras según su tipo, este Grupo tiene como misión prestar servicios de información bibliográfica a los usuarios con calidad y efectividad ofreciendo material pertinente con el fin de apoyar la enseñanza y el aprendizaje a través del Proceso de Gestión de Bibliotecas (López, 2010). La gestión de recursos humanos y de las tecnologías de la información son dos características muy importantes en la gestión del conocimiento, para el caso del Grupo de Bibliotecas e Información de la UPTC seccional Tunja se investigó y analizó sobre los aspectos positivos y negativos de la gestión del conocimiento y sus respectivas características.

\section{METODOLOGÍA}

El enfoque de la Investigación es cuantitativo debido a que se empleó la recolección de datos a través de la selección de una muestra de diez (10) personas entre funcionarios y becarios del Grupo de Bibliotecas e Información de la Universidad Pedagógica y Tecnológica de Colombia, se hizo uso de medición numérica y análisis estadístico, para establecer patrones de comportamiento y probar las teorías planteadas (Hernández et al., 2008).

El diseño de la presente investigación es de tipo no experimental y se realizó con el fin de determinar el impacto y la aplicación de la Gestión del Conocimiento en el Grupo de Bibliotecas e Información de la UPTC.

La técnica e instrumento para la recolección de datos utilizada fue la encuesta. La escala para medir las cinco (5) posibles alternativas de respuesta que se empleó se muestra en la Tabla 2: Siempre con un valor de muy alto, Casi Siempre, con un valor de alto, Ocasionalmente con un valor de medio, Casi Nunca con un valor de bajo y Nunca con un valor de muy bajo.

Las encuestas son instrumentos de investigación descriptiva que precisan identificar a priori las preguntas a realizar (Trespalacios et al., 2005). Dentro de esta investigación se selecciona una muestra de la población, se especifican las respuestas y se procede a determinar el método de análisis adecuado.

\section{Tabla 2}

Escala de medida

\begin{tabular}{|c|c|}
\hline Escala de medida & Valor \\
\hline Siempre & Muy alto \\
\hline Casi siempre & Alto \\
\hline Ocasionalmente & Medio \\
\hline Casi nunca & Bajo \\
\hline Nunca & Muy bajo \\
\hline
\end{tabular}

Fuente: Autores 


\section{ENCUESTA APLICADA A LA MUESTRA EN ESTUDIO (MODELO)}

\section{Gestión del conocimiento aplicado al de Bibliotecas e Información de la UPTC seccional Tunja}

Cargo: Funcionario

Becario

Según sus conocimientos del sistema de clasificación Bibliotecaria Dewey y teniendo en cuenta el cargo que desempeña en el Sistema de Bibliotecas e Información de la UPTC seccional Tunja, por favor responda la siguiente encuesta:

- Gestión de la novedad del conocimiento

1. Adquiere sus conocimientos con respecto a la bibliotecología por medio de fuentes confiables como capacitaciones, lecturas e interacción con otras personas del medio.

- Siempre

- Casi siempre

- Ocasionalmente

- Casi nunca

- Nunca

2. ¿Se adapta con facilidad a los posibles cambios que se presentan frente a los conocimientos que ya posee en su área?

- Siempre

- Casi siempre

- Ocasionalmente

- Casi nunca

- Nunca

- Gestión de la transformación del conocimiento

3. ¿Trasfiere los conocimientos que posee en el cargo que desempeña en el Grupo de Bibliotecas e Información de la UPTC a otras personas o usuarios?

- Siempre

- Casi siempre

- Ocasionalmente

- Casi nunca

- Nunca 
4. ¿Socializa las experiencias que vive en el cargo que desempeña en su área de trabajo para construir nuevo conocimiento?

- Siempre

- Casi siempre

- Ocasionalmente

- Casi nunca

- Nunca

- Gestión de la difusión y aplicación del conocimiento

5. ¿Con qué frecuencia realiza actividades que contribuyen al dialogo proactivo, abierto y dinámico para transmitir sus conocimientos a otras persona del área de trabajo?

- Siempre

- Casi siempre

- Ocasionalmente

- Casi nunca

- Nunca

6. Participa en acciones innovadoras que vinculen sus capacidades de aprendizaje y le aporten nuevos conocimientos en su área de trabajo

- Siempre

- Casi siempre

- Ocasionalmente

- Casi nunca

- Nunca

\section{Técnica para analizar los datos}

Antes de empezar es necesario tener claro que la presente investigación se realizó evaluando tres (3) aspectos esenciales:

- Gestión de la novedad del conocimiento

- Gestión de la transformación del conocimiento

- Gestión de la difusión y aplicación del conocimiento

Estos tres aspectos se tomaron como referencia de las investigaciones llamadas "Gestión del Conocimiento en las Bibliotecas del siglo XXI" (Shanhong, 2000) y "Gestión del conocimiento en institutos universitarios de tecnología" (Villamizar \& Weffer, 2011), donde se enfoca la gestión del conocimiento en bibliotecas e institutos Universitarios como es el caso de estudio. Además, se evaluaron dos (2) grupos de personas que hacen parte de la muestra de investigación dentro de los cuales están becarios y funcionarios (Tabla 3 ).

Para procesar los datos recolectados por medio de la encuesta y teniendo en cuenta los tres aspectos esenciales en el proceso del conocimiento se procedió a organizar los resultados futuros en gráficos de distribución porcentual debido a que la investigación es de naturaleza cuantitativa. Por otro lado, la información se muestra en tablas que son analizadas con cada uno de los aspectos evaluados, para proceder a discutir resultados, plantear estrategias y dar conclusiones. 
Tabla 3

Muestra de población Grupo de Bibliotecas e Información UPTC

\begin{tabular}{|c|c|c|c|c|}
\hline \multirow{2}{*}{$\begin{array}{c}\mathrm{N}^{\circ} \\
\text { de muestra }\end{array}$} & \multirow[b]{2}{*}{ Lugar en estudio } & \multicolumn{2}{|c|}{ Cargo } & \multirow[b]{2}{*}{ Total } \\
\hline & & Becario & Funcionario & \\
\hline 1 & \multirow{10}{*}{$\begin{array}{c}\text { Grupo de Bibliotecas e } \\
\text { Información de la UPTC seccional } \\
\text { Tunja }\end{array}$} & $\mathrm{X}$ & & 1 \\
\hline 2 & & $\mathrm{X}$ & & 1 \\
\hline 3 & & $\mathrm{X}$ & & 1 \\
\hline 4 & & $\mathrm{X}$ & & 1 \\
\hline 5 & & $\mathrm{X}$ & & 1 \\
\hline 6 & & & $\mathrm{X}$ & 1 \\
\hline 7 & & & $\mathrm{X}$ & 1 \\
\hline 8 & & & $\mathrm{X}$ & 1 \\
\hline 9 & & & $\mathrm{X}$ & 1 \\
\hline 10 & & & $\mathrm{X}$ & 1 \\
\hline & Total muestra & & & 10 \\
\hline
\end{tabular}

Fuente: Autores

\section{Análisis de los datos}

\section{Tabla 4}

Gestión de la novedad del conocimiento (becarios)

\begin{tabular}{|l|c|c|c|c|c|}
\hline $\begin{array}{r}\text { Opción de } \\
\text { respuesta }\end{array}$ & $\begin{array}{c}\text { Siempre } \\
(\%)\end{array}$ & $\begin{array}{c}\text { Casi siempre } \\
(\%)\end{array}$ & $\begin{array}{c}\text { Ocasionalmente } \\
(\%)\end{array}$ & $\begin{array}{c}\text { Casi nunca } \\
\text { Î) } \\
\text { Para evaluar }\end{array}$ & $\begin{array}{c}\text { Nunca } \\
(\%)\end{array}$ \\
\hline $\begin{array}{l}\text { Adquisición de cono- } \\
\text { cimiento por medio de } \\
\text { fuentes confiables }\end{array}$ & $60 \%$ & $40 \%$ & $0 \%$ & $0 \%$ & $0 \%$ \\
\hline $\begin{array}{l}\text { Adaptabilidad a cambios } \\
\text { en el conocimiento }\end{array}$ & $60 \%$ & $40 \%$ & $0 \%$ & $0 \%$ & $0 \%$ \\
\hline
\end{tabular}

Fuente: Autores 
En la Tabla 4 se puede observar que el $60 \%$ de los becarios del Grupo de Bibliotecas e Información adquieren sus conocimientos por medio de fuentes confiables que fomentan su capacidad analítica e interpretativa frente a las situaciones del área de trabajo, por otro lado el $40 \%$ de los becarios expresó que casi siempre obtiene la información de fuentes confiables, pero muchas veces se remiten a información de dudosa procedencia. En esta Tabla también se evalúa otro ítem llamado adaptabilidad a los cambios, donde se observa que el $60 \%$ de los becarios tienen la facilidad para adecuarse a los cambios que pueden ocurrir frente a su conocimiento y el $40 \%$ de la misma población tiene algunas dificultades para acomodarse a los cambios.

En el aspecto general de la Gestión de la novedad de conocimiento evaluado en la Tabla 4, se puede ver que la mayoría de los becarios adquieren nuevo conocimiento de fuentes confiables continuamente y se adaptan a los cambios que puede presentar la información ya adquirida (Figura 1).

\section{Figura 1}

Gestión de la novedad del conocimiento (becarios)
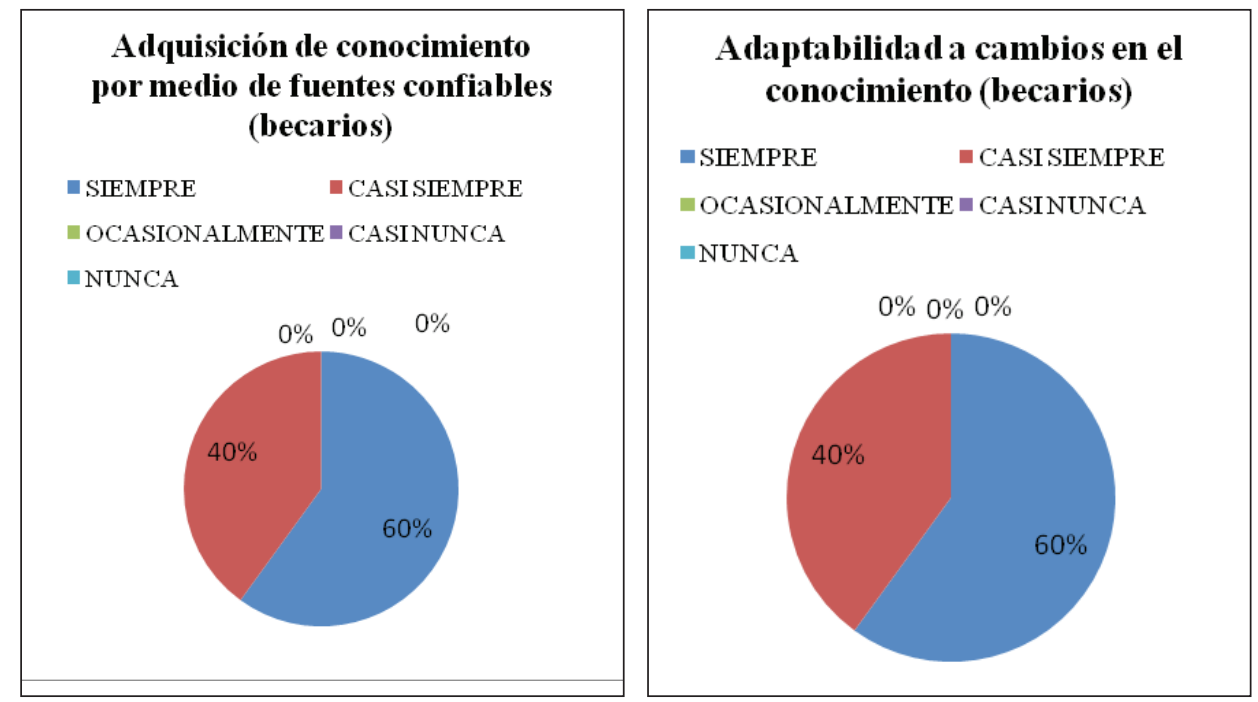

Fuente: Autores

Tabla 5

Gestión de la novedad del conocimiento (funcionarios)

\begin{tabular}{|l|c|c|c|c|c|}
\hline \multicolumn{1}{|c|}{ Opción de respuesta } & $\begin{array}{c}\text { Siempre } \\
\text { I\% })\end{array}$ & $\begin{array}{c}\text { Casi } \\
\text { siempre } \\
(\%)\end{array}$ & $\begin{array}{c}\text { Ocasionalmente } \\
(\%)\end{array}$ & $\begin{array}{c}\text { Casi } \\
\text { nunca } \\
(\%)\end{array}$ & $\begin{array}{c}\text { Nunca } \\
(\%)\end{array}$ \\
\hline $\begin{array}{l}\text { Adquisición de cono- } \\
\text { cimiento por medio de } \\
\text { fuentes confiables }\end{array}$ & $40 \%$ & $60 \%$ & $0 \%$ & $0 \%$ & $0 \%$ \\
\hline $\begin{array}{l}\text { Adaptabilidad a cambios } \\
\text { en el conocimiento }\end{array}$ & $20 \%$ & $80 \%$ & $0 \%$ & $0 \%$ & $0 \%$ \\
\hline
\end{tabular}

Fuente: Autores 
En relación con la Tabla 5, se observó que el 40\% de los funcionarios del Grupo de Bibliotecas e Información de la UPTC, adquiere su conocimiento por medio de fuentes confiables aumentando así sus habilidades y capacidades frente a los desafíos presentados en el desempeño de su cargo, mientras que el $60 \%$ de ellos expresó que casi siempre adquiere sus conocimientos de fuentes confiables, permitiéndole desarrollar de manera importante sus competencias contribuyendo así a mejorar el desempeño laboral y brindar un mejor servicio a los usuarios de la Biblioteca.

En la Tabla también se evaluó la capacidad de adaptabilidad al cambio en el conocimiento por parte de los funcionarios del Grupo de Bibliotecas e Información, en lo cual se observó que el $20 \%$ de ellos posee tal capacidad lo que les permite desenvolverse de manera ágil en cualquier entorno o situación presentada, mientras que el $80 \%$ está un poco más reacia a la aceptación de dichos cambios.

En relación con el aspecto sobre la Gestión de la Novedad del conocimiento, se puede ver que en la mayoría de los casos, los funcionarios no buscan fuentes confiables a la hora de incrementar su conocimiento y de igual manera, no se adaptan con facilidad al cambio en el entorno, lo que dificulta su desempeño laboral (Figura 2).

\section{Figura 2}

Gestión de la novedad del conocimiento (funcionarios)

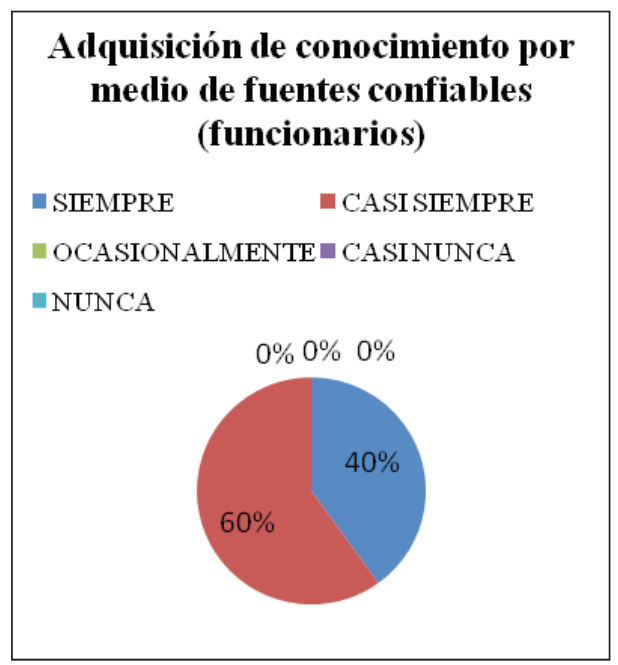

Adaptabilidad a cambios en el conocimiento (funcionarios)

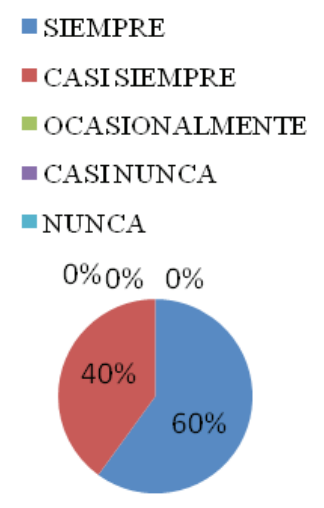

Fuente: Autores 


\section{Tabla 6}

Gestión de la transformación del conocimiento (becarios)

\begin{tabular}{|l|c|c|c|c|c|}
\hline $\begin{array}{r}\text { Opción de } \\
\text { respuesta }\end{array}$ & $\begin{array}{c}\text { Siempre } \\
(\%)\end{array}$ & $\begin{array}{c}\text { Casi } \\
\text { siempre } \\
(\%)\end{array}$ & $\begin{array}{c}\text { Ocasionalmente } \\
(\%)\end{array}$ & $\begin{array}{c}\text { Casi } \\
\text { nunca } \\
(\%)\end{array}$ & $\begin{array}{c}\text { Nunca } \\
(\%)\end{array}$ \\
\hline $\begin{array}{l}\text { Ítem } \\
\text { Para evaluar }\end{array}$ & $80 \%$ & $0 \%$ & $20 \%$ & $0 \%$ & $0 \%$ \\
\hline $\begin{array}{l}\text { Transferencia de } \\
\text { conocimiento }\end{array}$ & $40 \%$ & $40 \%$ & $20 \%$ & $0 \%$ & $0 \%$ \\
\hline $\begin{array}{l}\text { Socialización de } \\
\text { experiencias }\end{array}$ & &
\end{tabular}

Fuente: Autores

Al describir los resultados de la Tabla 6, se puede ver como el $80 \%$ de los becarios transfiere su conocimiento a otras personas y usuarios de forma constante, pues el valor que arrojó el ítem evaluado tiene un alto porcentaje. Por otra parte, el $20 \%$ de la población realiza ocasionalmente el ejercicio de transferencia de conocimiento lo que altera en parte la transformación del mismo.

En cuanto al ítem de socialización se pudo notar que un $40 \%$ de la población siempre socializa sus experiencias en el área de trabajo, otro $40 \%$ casi siempre comparte sus experiencias y un $20 \%$ ocasionalmente participa en el hecho de transmitir sus vivencias. Como tal la gestión de la transformación del conocimiento se da de manera fuerte en el hecho de transferencia del mismo, pero de manera muy débil al momento de socializar las experiencias vividas en el área de trabajo, lo que dificulta el hecho del aprendizaje basado en la práctica (Figura 3).

\section{Figura 3}

Gestión de la transformación del conocimiento (becarios)
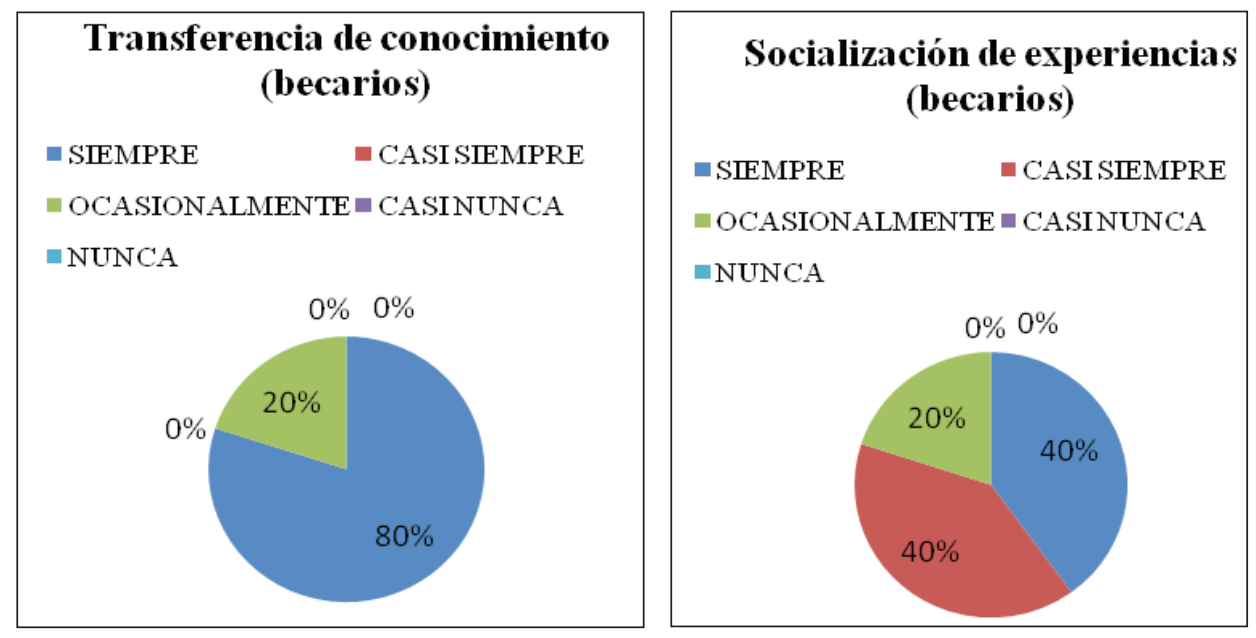

Fuente: Autores 
Tabla 7

Gestión de la transformación del conocimiento (funcionarios)

\begin{tabular}{|l|c|c|c|c|c|}
\hline $\begin{array}{r}\text { Opción de } \\
\text { respuesta }\end{array}$ & $\begin{array}{c}\text { Siempre } \\
(\%)\end{array}$ & $\begin{array}{c}\text { Casi } \\
\text { siempre } \\
(\%)\end{array}$ & $\begin{array}{c}\text { Ocasionalmente } \\
(\%)\end{array}$ & $\begin{array}{c}\text { Casi } \\
\text { nunca } \\
(\%)\end{array}$ & $\begin{array}{c}\text { Nunca } \\
(\%)\end{array}$ \\
\hline $\begin{array}{l}\text { Ítem } \\
\text { Para evaluar }\end{array}$ & $60 \%$ & $40 \%$ & $0 \%$ & $0 \%$ & $0 \%$ \\
\hline $\begin{array}{l}\text { Transferencia de } \\
\text { conocimiento }\end{array}$ & $20 \%$ & $40 \%$ & $40 \%$ & $0 \%$ & $0 \%$ \\
\hline $\begin{array}{l}\text { Socialización de } \\
\text { experiencias }\end{array}$ & & & $0 \%$ & \\
\hline
\end{tabular}

Fuente: Autores

En la Tabla 7, se observó que el 70\% de los funcionarios están siempre abiertos a transferir sus conocimientos en el área de trabajo a los usuarios de la biblioteca para hacer más ágil y rápida la búsqueda de ejemplares, por otro lado, el $40 \%$ no siempre está dispuesto a realizar dicha actividad. Así mismo, sólo el $20 \%$ de ellos está abierto a socializar experiencias en su campo de trabajo con los demás compañeros, mientras que el $40 \%$ casi siempre u ocasionalmente lo hace.

En el aspecto de la Gestión de Transformación del conocimiento, se observó que hay un gran porcentaje de funcionarios dispuestos a compartir o transferir sus conocimientos a compañeros o usuarios para de una u otra manera mejorar el servicio que se presta, por otro lado, se ve que no siempre están abiertos a socializar las diferentes experiencias evidenciadas en el desempeño de su cargo (Figura 4).

\section{Figura 4}

Gestión de la transformación del conocimiento (funcionarios)
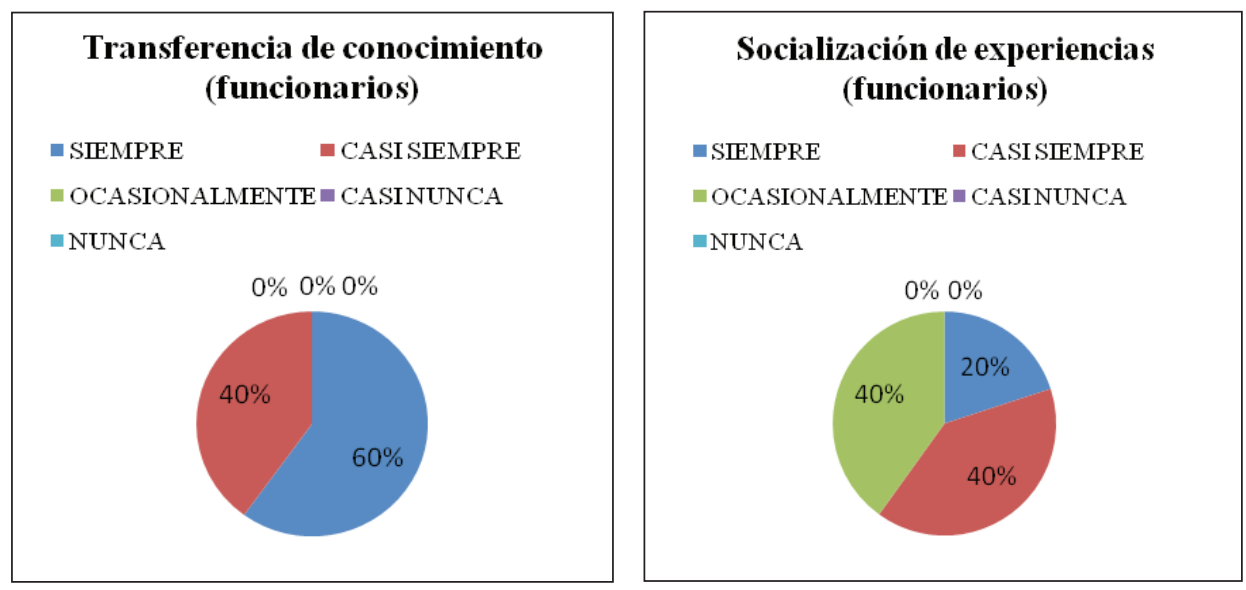

Fuente: Autores 


\section{Tabla 8}

Gestión de la Difusión y Aplicación del Conocimiento (becario)

\begin{tabular}{|l|c|c|c|c|c|}
\hline $\begin{array}{r}\text { Opción de } \\
\text { respuesta }\end{array}$ & $\begin{array}{c}\text { Siempre } \\
(\%)\end{array}$ & $\begin{array}{c}\text { Casi } \\
\text { siempre } \\
(\%)\end{array}$ & $\begin{array}{c}\text { Ocasionalmente } \\
(\%)\end{array}$ & $\begin{array}{c}\text { Casi } \\
\text { nunca } \\
(\%)\end{array}$ & $\begin{array}{c}\text { Nunca } \\
(\%)\end{array}$ \\
\hline $\begin{array}{l}\text { Ítem } \\
\text { Para evaluar }\end{array}$ & $40 \%$ & $60 \%$ & $0 \%$ & $0 \%$ & $0 \%$ \\
\hline $\begin{array}{l}\text { Actividades para } \\
\text { transmitir nuevo } \\
\text { conocimiento }\end{array}$ & $0 \%$ & $60 \%$ & $40 \%$ & $0 \%$ & $0 \%$ \\
\hline $\begin{array}{l}\text { Participación } \\
\text { en acciones } \\
\text { innovadoras }\end{array}$ & & & & \\
\hline
\end{tabular}

Fuente: Autores

La Tabla 8 muestra el comportamiento de los becarios frente a las actividades para transmitir el conocimiento, donde el $40 \%$ de la población afirmó que dentro de sus tareas incluye el diálogo proactivo, abierto y dinámico para difundir su conocimiento en cambio el $60 \%$ no lo hace de forma habitual. Por otro lado, el $60 \%$ de los becarios casi siempre realiza actividades innovadoras para vincular la capacidad de aprendizaje pero el $40 \%$ restante lo realiza ocasionalmente trayendo como posible consecuencia que no se aporten nuevos conocimientos en el área de trabajo.

Como tal se puede ver que en el aspecto de Gestión de la difusión y aplicación del conocimiento se realizan pocas actividades para establecer una comunicación activa y directa, además la población encuestada con poca frecuencia participa en actividades innovadoras que le aporten nuevos conocimientos (Figura 5).

Figura 5

Gestión de la difusión y aplicación del conocimiento (becario)
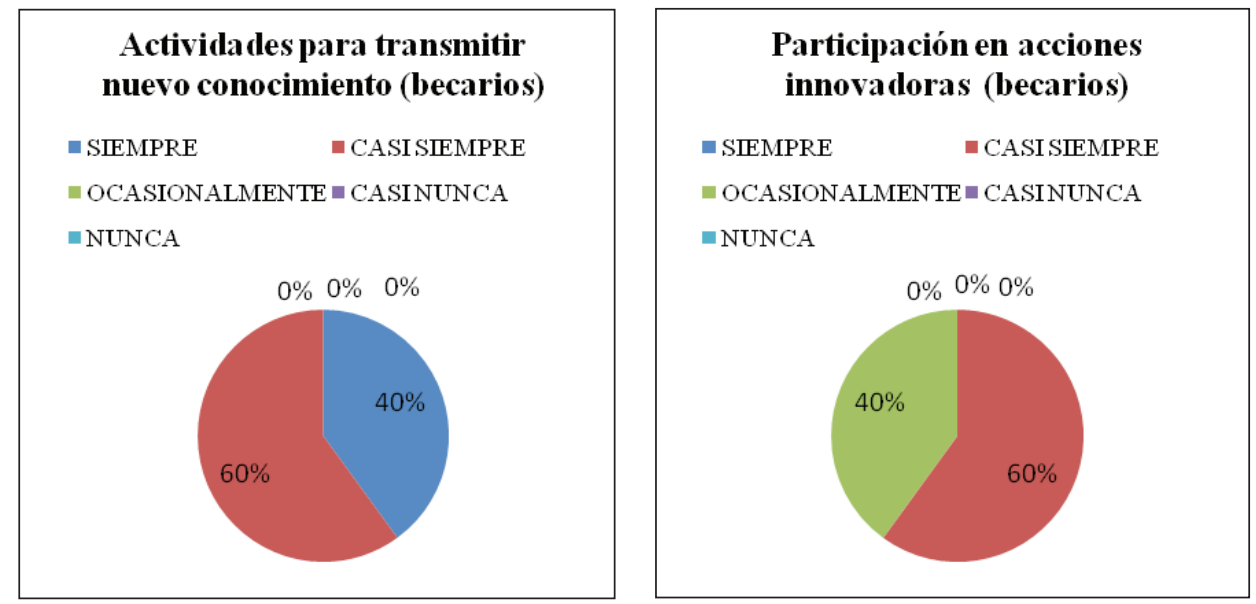

Fuente: Autores 
Tabla 9

Gestión de la Difusión y Aplicación del Conocimiento (funcionario)

\begin{tabular}{|l|c|c|c|c|c|}
\hline $\begin{array}{r}\text { Opción de } \\
\text { respuesta }\end{array}$ & $\begin{array}{c}\text { Siempre } \\
(\%)\end{array}$ & $\begin{array}{c}\text { Casi } \\
\text { siempre } \\
(\%)\end{array}$ & $\begin{array}{c}\text { Ocasionalmente } \\
(\%)\end{array}$ & $\begin{array}{c}\text { Casi } \\
\text { nunca } \\
(\%)\end{array}$ & $\begin{array}{c}\text { Nunca } \\
(\%)\end{array}$ \\
\hline $\begin{array}{l}\text { Actividades para } \\
\text { Paransmitir nuevo } \\
\text { conocimiento }\end{array}$ & $0 \%$ & $0 \%$ & $100 \%$ & $0 \%$ & $0 \%$ \\
\hline $\begin{array}{l}\text { Participación } \\
\text { en acciones } \\
\text { innovadoras }\end{array}$ & $40 \%$ & $0 \%$ & $60 \%$ & $0 \%$ & $0 \%$ \\
\hline
\end{tabular}

Fuente: Autores

Con respecto a la Tabla 9, se observó que el $100 \%$ de los funcionarios solo ocasionalmente están dispuestos a desarrollar actividades que permitan transmitir nuevo conocimiento. Por otra parte, el $40 \%$ de ellos participa siempre en actividades innovadoras que fortalecen la gestión de conocimiento en el Grupo de Bibliotecas e Información, mientras el 60\% de los funcionarios solo ocasionalmente contribuyen con actividades para la mejora del sistema.

En el aspecto de la Gestión de la difusión y aplicación del conocimiento, se determinó que no siempre se está dispuesto a llevar a cabo actividades que contribuyan a transmitir el conocimiento a los usuarios para desarrollar una labor más eficiente y no siempre se participa en actividades de innovación y fortalecimiento del conocimiento (Figura 6).

\section{Figura 6}

Gestión de la difusión y aplicación del conocimiento (funcionario)
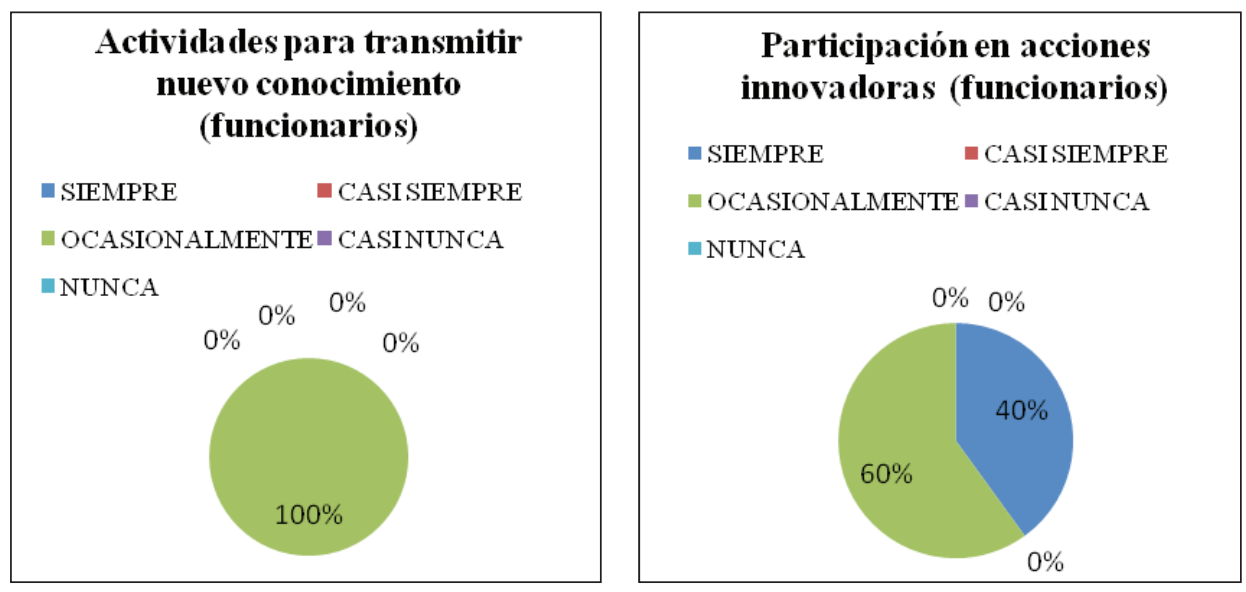

Fuente: Autores 


\section{RESULTADOS}

Al realizar un análisis general de la información obtenida en la presente investigación y recordando los tres aspectos evaluados se pueden ver algunas virtudes y falencias en la labor del conocimiento dentro de Grupo de Bibliotecas e Información de la UPTC. La muestra tomada fue dividida en dos (2) grupos, el primero los becarios y el segundo los funcionarios. Esto se hizo con el fin de medir los niveles de conocimientos de cada uno de ellos y ver los posibles pros y contras del mismo dentro de su área de trabajo.

Evaluando el primer aspecto llamado Gestión de la novedad del conocimiento se pudo observar que en la muestra de becarios más de la mitad de las personas encuestadas $(60 \%)$ respondió que siempre adquiere sus conocimientos por medio de fuentes confiables y se adapta con facilidad a los cambios del mismo. Teniendo en cuenta lo anterior se puede ver una tendencia de valor muy alto para este grupo. Sin embargo, el restante de la muestra no tiene un valor muy alto lo que quiere decir que se encuentra falencia en el ámbito de obtención del conocimiento dentro de este grupo.

Así que examinando el mismo aspecto de la novedad del conocimiento pero con la población de funcionarios, se pudo ver como más de la mitad de la muestra $(60 \%)$ casi siempre adquiere los conocimientos de fuentes confiables. En cuanto a la adaptabilidad a los cambios la muestra reflejo que casi siempre (80\%) los funcionarios se adaptan a transformaciones (Tabla 10).

Se puede ver en este último suceso que la población de funcionarios presenta algunas dificultades a la hora de ajustarse a los cambios del conocimiento y que a su vez la información que poseen no se toma con frecuencia de fuentes creíbles.

\section{Tabla 10}

Resultados obtenidos en la gestión de la novedad del conocimiento

\begin{tabular}{|c|c|c|c|}
\hline Muestra & Aspecto evaluado & Muestra de mayor peso & valor \\
\hline \multirow{2}{*}{ Becario } & Adquisición & Siempre $(60 \%)$ & Muy alto \\
\cline { 2 - 4 } & Adaptabilidad & Siempre $(60 \%)$ & Muy alto \\
\hline \multirow{2}{*}{ Funcionario } & Adquisición & Casi siempre $(60 \%)$ & Alto \\
\cline { 2 - 4 } & Adaptabilidad & Casi siempre $(80 \%)$ & Alto \\
\hline
\end{tabular}

Fuente: Autores

Estudiando el segundo aspecto conocido como la Gestión de la transformación del conocimiento, en la muestra de becarios se pudo ver un valor muy alto $(80 \%)$ hacia la transferencia del conocimiento, pero valores medianamente altos (40\%) en el ítem de socialización del conocimiento. Al ver las muestras con mayor peso se puede observar que los becarios transmiten sus 
conocimientos con facilidad a las personas de su área de trabajo, pero se ven en gran dificultad al momento de socializar las experiencias adquiridas.

Por otra parte en el grupo de funcionarios se pudo visualizar un valor muy alto $(60 \%)$ hacia la transferencia de conocimiento y valores medianamente altos $(40 \%)$ con el tema de socialización de vivencias en el área de trabajo lo que representa deficiencias muy marcadas en este ítem. El valor mostrado por los funcionarios a la hora de transmitir la información tiene algunas falencias en comparación al grupo de becarios evaluados (Tabla 11).

\section{Tabla 11}

Resultados obtenidos en la Gestión de la transformación del conocimiento

\begin{tabular}{|c|c|c|c|}
\hline Muestra & Aspecto evaluado & Muestra de mayor peso & valor \\
\hline \multirow{3}{*}{ Becario } & Transferencia & Siempre $(80 \%)$ & Muy alto \\
\cline { 2 - 4 } & Socialización & Siempre $(40 \%)$ & Muy alto \\
\cline { 3 - 4 } & & Casi siempre $(40 \%)$ & Alto \\
\hline \multirow{3}{*}{ Funcionario } & Transferencia & Siempre $(60 \%)$ & Muy alto \\
\cline { 2 - 4 } & Socialización & Casi siempre $(40 \%)$ & Alto \\
\cline { 3 - 4 } & & Ocasionalmente $(40 \%)$ & Medio \\
\hline
\end{tabular}

Fuente: Autores

Por último, analizando el aspecto de Gestión de la difusión y aplicación del conocimiento en el grupo de becarios se pudo contemplar un valor alto $(60 \%)$ en el ítem de actividades para transmisión de conocimiento lo que demuestra que existen debilidades con respecto al dialogo proactivo y dinámico en el área de trabajo.

En el ítem de acciones innovadoras se pudo observar el mismo valor alto $(60 \%)$ a la hora de vincular las capacidades de aprendizaje y aporte de nuevos conocimientos.

En el grupo de funcionarios las actividades de transferencia arrojaron un valor medio (100\%) lo que se puede ver como una falencia enorme, esto quiere decir que esta parte de la muestra ocasionalmente se ve dispuesta a interactuar mediante el diálogo dinámico y proactivo con las personas del área de trabajo. Por otro lado se puede ver también un valor medio (60\%) en la participación de acciones innovadoras, en este punto se observan las dificultades de este grupo con respecto a la comunicación y divulgación del conocimiento (Tabla 12). 
Tabla 12

Resultados obtenidos en la Gestión de la difusión y aplicación del conocimiento

\begin{tabular}{|l|c|c|c|}
\hline \multicolumn{1}{|c|}{ Muestra } & Aspecto evaluado & Muestra de mayor peso & valor \\
\hline \multirow{2}{*}{ Becario } & Actividades & Casi siempre $(60 \%)$ & Alto \\
\cline { 2 - 4 } & Acciones innovadoras & Casi siempre $(60 \%)$ & Alto \\
\hline \multirow{2}{*}{ Funcionario } & Actividades & Ocasionalmente $(100 \%)$ & Medio \\
\cline { 2 - 4 } & Acciones innovadoras & Ocasionalmente $(60 \%)$ & Medio \\
\hline
\end{tabular}

Fuente: Autores

\section{DISCUSIÓN Y CONCLUSIÓN}

Según los tres aspectos evaluados en el desarrollo de la investigación es importante identificar por qué se presentan falencias en algunos de los puntos evaluados con el fin de crear acciones que fomenten de forma adecuada la gestión del conocimiento.

1. Gestión de la novedad del conocimiento:

a. Adquisición de conocimiento por medio de fuentes confiables: Observando los resultados generales de la encuesta aplicada según lo evidenciado en la Tabla 10, la adquisición de conocimiento de fuentes no confiables es un fenómeno que ataca en su mayoría a la población de funcionarios debido al desconocimiento en algunas ocasiones del manejo de las TIC'S lo que les impide adquirir información actualizada y confiable.

b. Adaptabilidad a posibles cambios: En este punto la población de becarios toma una gran ventaja pues según los resultados visualizados son los que siempre se adaptan con mayor facilidad a los cambios, pero esto es un suceso normal pues a medida que pasan los años se tiene más miedo a la inestabilidad o a las transiciones trascendentales.

2. Gestión de la transformación del conocimiento:

a. Transferencia de conocimiento: Al observar la Tabla 11 es posible ver como la mayoría de la población de becarios realizan procesos de transferencia de conocimiento, hecho que resulta ser indispensable para que los nuevos saberes sean cultivados en diversas personas. Por el lado, de los funcionarios se puede notar un porcentaje un poco más bajo para transferir el conocimiento situación que se puede presentar por la distancia que existe entre algunos funcionarios y personas del común.

b.Socialización de experiencias: Este es un punto en común entre la población de funcionarios y becarios, y se presenta en su mayoría por el miedo al rechazo, a los comentarios que puedan derivarse al contar experiencias de errores o problemas que se presentan en el área de trabajo, para este hecho es imprescindible crear un ambiente de confianza y armonía para que se puedan compartir vivencias que sirvan a otras personas como punto de referencia. 
3. Gestión de la difusión y aplicación del conocimiento:

a.Actividades para difundir el conocimiento: Los becarios al pertenecer a la población de estudiantes realizan a diario actividades que les exigen replicar el conocimiento a diferencia de los funcionarios quienes están mecanizados en algunas ocasiones a realizar actividades de rutina, situación que se observa en los porcentajes generales de la Tabla 12 .

b. Acciones innovadoras: Este aspecto se centra en los actos que involucren creación y descubrimiento de nuevo conocimiento, parámetro que resulta elemental en las bibliotecas donde en la actualidad la gente no asiste con la misma frecuencia. Para este punto los becarios expresaron su agrado por participar en acciones innovadoras que generen nuevas competencias, situación en la cual los funcionarios no mostraron las mismas ganas debido al miedo por adecuarse a reformas que se presenten en el área de trabajo.

\section{Acciones que fomenten la gestión del conocimiento}

El conocimiento se crea a través de un proceso de interacción continuo y dinámico entre el conocimiento tácito y el explícito, de carácter personal o colectivo, que incluye momentos de socialización, exteriorización, combinación e interiorización. La interactividad es el eje a través del cual se va construyendo el conocimiento. No se reduce a un intercambio de información, sino que supone una verdadera negociación entre los participantes, produciéndose un diálogo fructífero al compartir ideas, lo que genera aprendizaje activo y extiende los propios puntos de vista al considerarlos desde diferentes perspectivas (Gairín, 2007).

Analizando el concepto dado anteriormente y partiendo de la importancia y necesidad de comunicar de forma adecuada los conocimientos que se adquieren en el área de trabajo se realiza un estudio de las fortalezas y debilidades en forma general para luego proporcionar estrategias que se encaminen a la apropiada gestión del conocimiento.
Teniendo en cuenta lo expresado en la Tabla 13 y la evaluación de resultados se propone:

- Incentivar y promover la adquisición correcta y de fuentes confiables de la información para que de esta forma el grupo tenga los conocimientos adecuados y necesarios que exige el área de trabajo.

- Realizar simulacros en los que se vean involucrados cambios en el área de trabajo con el fin de adaptar a las personas a las posibles transformaciones que puede sufrir el conocimiento.

- Teniendo en cuenta que se ha realizado de forma apropiada la adquisición del conocimiento se debe impulsar a las personas del área de trabajo para que transfieran la información que ya poseen a personas novatas o a usuarios del Grupo de Bibliotecas e Información de la UPTC.

- El aspecto de socialización fue uno de los más débiles en forma general en la muestra estudiada por tal razón se plantea como solución talleres vivenciales donde las personas compartan las experiencias positivas y negativas en el grupo de trabajo para que de ésta forma todas las personas aprendan de las situaciones que los demás han vivido.

- Para que exista una difusión del conocimiento se debe impeler a la comunidad a que realice actividades que contribuyan al diálogo proactivo, abierto y directo de forma que se pueda transmitir la información en el grupo de trabajo.

- Es de vital importancia que las personas participen en acciones innovadoras del conocimiento, por tal razón, es necesario que se busquen espacios que fomenten la vinculación de las capacidades de aprendizaje y de aporte de nuevos conocimientos en el grupo de trabajo.

En el aspecto de la Gestión de la novedad del conocimiento, se ha podido observar que la mayoría de los funcionarios y becarios del Grupo de Bibliotecas e Información de la UPTC tienen en cuenta la importancia de la adquisición de nuevos conocimientos mediante el uso de fuentes confiables, permitiendo así que lo aprendido sea de calidad y completamente válido para no generar posibles confusiones al momento de prestar el servicio a la comunidad usuaria de la biblioteca. 
Tabla 13

Análisis de los aspectos generales frente a las virtudes y falencias

\begin{tabular}{|c|c|c|}
\hline \multicolumn{3}{|c|}{ Becarios } \\
\hline Aspecto evaluado & Virtudes & Falencias \\
\hline $\begin{array}{l}\text { Adquisición de } \\
\text { conocimiento por } \\
\text { medio de fuentes } \\
\text { confiables }\end{array}$ & $\begin{array}{l}\text { Parte de la muestra adquiere } \\
\text { el conocimiento de fuentes } \\
\text { confiables como lecturas, } \\
\text { capacitaciones e interacción } \\
\text { con personal especializado. }\end{array}$ & $\begin{array}{l}\text { Otras personas de la muestra } \\
\text { aún consulta información } \\
\text { que puede no ser verídica } \\
\text { afectando la obtención de } \\
\text { nuevo conocimiento. }\end{array}$ \\
\hline $\begin{array}{l}\text { Adaptabilidad } \\
\text { a cambios en el } \\
\text { conocimiento }\end{array}$ & $\begin{array}{l}\text { Una fracción de la muestra } \\
\text { se puede acomodar fácil- } \\
\text { mente a los cambios que se } \\
\text { presentan frente a los cono- } \\
\text { cimientos que ya poseen. }\end{array}$ & $\begin{array}{l}\text { Parte de la muestra estu- } \\
\text { diada aún teme al cambio } \\
\text { y transformación del co- } \\
\text { nocimiento que ya tienen } \\
\text { contenido en su ser. }\end{array}$ \\
\hline $\begin{array}{l}\text { Transferencia de } \\
\text { conocimiento }\end{array}$ & $\begin{array}{l}\text { La mayoría de la muestra } \\
\text { encuestada transfiere sus } \\
\text { conocimientos a otras per- } \\
\text { sonas y usuarios en el área } \\
\text { de trabajo }\end{array}$ & $\begin{array}{l}\text { Un porcentaje pequeño de } \\
\text { la muestra no comunica } \\
\text { sus conocimientos a los } \\
\text { demás, lo que no permite } \\
\text { la transformación de la } \\
\text { información. }\end{array}$ \\
\hline $\begin{array}{l}\text { Socialización de } \\
\text { experiencias }\end{array}$ & $\begin{array}{l}\text { Una parte de la muestra } \\
\text { socializa sus experiencias } \\
\text { vividas en el cargo que } \\
\text { desempeña fomentando } \\
\text { seguridad a los nuevos } \\
\text { aspirantes. }\end{array}$ & $\begin{array}{l}\text { Se encuentran debilidades } \\
\text { en otra parte de la muestra } \\
\text { que no expresa a los demás } \\
\text { sus vivencias en el área de } \\
\text { trabajo, ya sea por miedo o } \\
\text { rechazo. }\end{array}$ \\
\hline $\begin{array}{l}\text { Actividades para } \\
\text { transmitir nuevo } \\
\text { conocimiento }\end{array}$ & $\begin{array}{l}\text { Gran parte de la muestra } \\
\text { ocasionalmente realiza ac- } \\
\text { tividades que incentiven } \\
\text { la comunicación dinámica } \\
\text { y fluida. }\end{array}$ & $\begin{array}{l}\text { Pocas de las personas per- } \\
\text { tenecientes a la muestra } \\
\text { contribuyen al diálogo diná- } \\
\text { mico y abierto en el grupo } \\
\text { de trabajo. }\end{array}$ \\
\hline $\begin{array}{l}\text { Participación } \\
\text { en acciones } \\
\text { innovadoras }\end{array}$ & $\begin{array}{l}\text { La mayoría de la muestra } \\
\text { estudiada de vez en cuando } \\
\text { hace parte de actividades } \\
\text { novedosas debido a la falta } \\
\text { de tiempo. }\end{array}$ & $\begin{array}{l}\text { Muy pocas personas reali- } \\
\text { zan acciones innovadoras } \\
\text { donde emplean sus capaci- } \\
\text { dades de aprendizaje }\end{array}$ \\
\hline
\end{tabular}

Fuente: Autores

Basándose en el mismo aspecto, se puede concluir que un alto porcentaje de becarios de la organización están dispuestos y preparados para asumir los nuevos retos y cambios que se presenten en el día a día durante el desarrollo de sus actividades dentro del Grupo, mientras que los funcionarios, quienes deberían estar más abiertos a dichos sucesos, no siempre están dispuestos a afrontarlos afectando su desempeño en el área de trabajo.

Es imperativo la implementación de un Sistema de Gestión del Conocimiento en el Grupo de Bibliotecas e Información de la UPTC seccional Tunja que 
contribuya a fortalecer las debilidades frente a los aspectos mencionados en la investigación, para fortalecer aquellos puntos débiles en los funcionarios principalmente, quienes se ven un poco reacios a los cambios, a la socialización y transferencia de los conocimientos propios para mejorar el servicio prestado por su área de trabajo.

La gestión del conocimiento permitirá al Grupo de Bibliotecas e Información de la UPTC seccional Tunja desarrollar un plan estratégico que contribuya a la mejora en la calidad de los servicios prestados por la organización, así mismo ayudará en el desarrollo de competencias profesionales adecuadas por parte de cada uno de sus funcionarios y trabajadores, haciendo que los servicios que allí se prestan puedan satisfacer de manera completa las necesidades de la comunidad Universitaria.

\section{REFERENCIAS BIBLIOGRÁFICAS}

Bonnet, N. (2005). La Gestión del conocimiento aplicado a los recursos de bibliotecas públicas. Recuperado de: http://hum.unne.edu.ar/revistas/ postgrado/revista5/articulos/bonnet.pdf

Davenport, T. H., \& Prusak, L. (2001). Conocimiento en Acción: Cómo las organizaciones manejan lo que saben. Prentice Hall.

Drucker, P. \& Toffler, A. (2011). Gestión del conocimiento: contexto y antecedentes. Recuperado de: http:// www.profesionalesdelconocimiento.com/profcon/ index.php?option $=$ com_content $\&$ view $=$ article \&id = 206:gestion-del-conocimiento-contexto-yantecedentes \&catid $=3$ :flash-noticioso\&Itemid $=60$
Gairín, J. (2007). Competencias para la gestión del conocimiento y el aprendizaje. Cuadernos de Pedagogía, 370, 24-27.

Gómez, D. (2007). Modelos para la creación y gestión del conocimiento: una aproximación teórica. Educar, (37), 25-39.

Hernández et al., (2008). Metodología de la investigación $3^{\text {a }} \mathrm{Ed}$.

López, A. (2010)Grupo de Bibliotecas UPTC información general. Recuperado de: http://www.uptc.edu. co/servicios/biblioteca/inf_general/

Polanyi, M., \& Sen, A. (1996). The tacit dimension (pp. 21-25). Gloucester, MA: Peter Smith.

Sánchez García de las Bayonas, S. (2007). Análisis de la gestión del conocimiento en las bibliotecas universitarias de la Comunidad Valenciana: propuesta de un modelo y acciones de mejora. Scire, 13(2).

Sandoval Yáñez, C. (2013). Propuesta para implementar un sistema de gestión del conocimiento que apoye el diseño de un curso online. Ingeniare. Revista chilena de ingeniería, 21 (3), 457-471.

Shanhong, T. (2000). Gestión del conocimiento en las bibliotecas del siglo XXI. In 66th IFLA Council and General Conference (pp. 13-18).

Tardón, E. (1999). Bibliotecas universitarias y gestión del conocimiento. El Profesional de la Información [en línea], 8 (4).

Trespalacios et al., (2005). Investigación de mercados: métodos de recogida y análisis de la información para la toma de decisiones en marketing. España: Editorial Paraninfo.

UPTC, (2005). Estatuto General, Acuerdo 066 del 25 de octubre de 2005. Recuperado de: http://www. uptc.edu.co/secretaria_general/estatutos/

Verde E., Blanco, C., \& Mata, E. (2005). Gestión del conocimiento como fuente innovadora para las bibliotecas del sector universitario.

Villamizar, Y. C. U., \& Weffer, R. V. (2011). Gestión del conocimiento en institutos universitarios de tecnología. Praxis, 7 (1). 